\title{
The nonlinear effects on the characteristics of gravity wave packets: dispersion and polarization relations
}

\author{
S.-D. Zhang, F. Yi, J.-F. Wang \\ Department of Space Physics, Wuhan University, Wuhan, 430072, P.R. China
}

Received: 24 January 2000 / Revised: 3 May 2000 / Accepted: 10 May 2000

\begin{abstract}
By analyzing the results of the numerical simulations of nonlinear propagation of three Gaussian gravity-wave packets in isothermal atmosphere individually, the nonlinear effects on the characteristics of gravity waves are studied quantitatively. The analyses show that during the nonlinear propagation of gravity wave packets the mean flows are accelerated and the vertical wavelengths show clear reduction due to nonlinearity. On the other hand, though nonlinear effects exist, the time variations of the frequencies of gravity wave packets are close to those derived from the dispersion relation and the amplitude and phase relations of wave-associated disturbance components are consistent with the predictions of the polarization relation of gravity waves. This indicates that the dispersion and polarization relations based on the linear gravity wave theory can be applied extensively in the nonlinear region.
\end{abstract}

Key words: Meteorology and atmospheric dynamics (middle atmosphere dynamics; waves and tides)

\section{Introduction}

It is extensively accepted that the propagation of gravity waves is one of the most important and frequent kinetic phenomena that occurs in the middle and upper atmosphere. A number of theoretical studies of gravity waves have emphasized the importance of gravity waves in transporting energy and momentum from lower atmosphere to the upper middle atmosphere (Lindzen, 1981; Holton, 1982, 1983; Fritts and Rastogi, 1985), which leads to the coupling of different layers in

Correspondence to: S.-D. Zhang

e-mail: zsd@whu.edu.cn atmosphere and plays an important role in energy balance in the global middle and upper atmosphere. Since the 1960s it is clearly understood that when a gravity wave packet propagates in the atmosphere, the background flow will be accelerated because of the transient nature of a wave packet, moreover, since the atmospheric density decreases exponentially with increasing height, upgoing gravity waves excited in the troposphere reach such great amplitudes near the mesosphere that they break and cause intensive turbulence (Lindzen, 1981; Fritts, 1984). These effects are considered to be significant for the formation of the global thermal and wind structure (Fritts, 1984).

Since the 1960s, various aspects of linear gravity wave theories have been extensively studied (Yeh and Liu, 1974). By ignoring all sources and loss processes, under the linear approximation, Hines (1960) worked out the dispersion and polarization relations of gravity waves in an isothermal atmosphere, this approach provides the most fundamental base for later studies of gravity waves. Because of the exponential increase of gravity wave-associated disturbance velocities with height, it is believed that the nonlinearity will strongly affect the propagation of gravity waves in the middle and upper atmosphere. Therefore after the 1980s, many authors have thrown light on the nonlinear effects of gravity waves and a number of valuable approaches (Lindzen, 1981; Fritts, 1984; Dewan and Good, 1986; Hines, 1991; Gardner, 1994) have been presented. However, all these approaches are carried out by making a crucial assumption: that all gravity waves, including those in the nonlinear circumstance, obey the polarization and dispersion relations of gravity waves.

Observation is one of the most important methods in studying gravity waves. However, any of the groundbased remote sensing facilities can only provide information of wave frequency and vertical wave number (Vincent and Fritts, 1987; Vincent 1994). The horizontal wave number is usually estimated by using the correlation analysis, or derived from the polarization and dispersion relations (Meek et al., 1985; Yamamoto et al., 1986; Manson, 1990; Tsuda et al., 1990). Therefore it is 
specially significant to examine quantitatively the validity of dispersion and polarization relations based essentially on the linear gravity-wave theory in the nonlinear region.

The numerical simulation is the most effective method of dealing with nonlinear problems. Since the 1980s, many authors have simulated the nonlinear process of gravity waves in the middle and upper atmosphere (e.g. Mobbs, 1987; Prusa, 1996; Zhang and Yi, 1999). In their numerical studies, although they discussed many nonlinear phenomena in the propagation of gravity waves, they did not examine the validity of dispersion and polarization relations in the nonlinear region. Walterscheid and Schubert (1990) simulated the nonlinear evolution of an upward propagating gravity wave by using a fully nonlinear and compressible model, in their study, they examined the intrinsic frequency and concluded that the wave approximately conserved its initial intrinsic frequency. In this work we present numerical simulations of two-dimensional nonlinear propagation of three individual gravity wave packets in a compressible atmosphere by using the full-implicit-continuousEulerian (FICE) scheme ( $\mathrm{Hu}$ and $\mathrm{Wu}, 1984)$. The numerical model and computational method are introduced in Sect. 2. Considering three upgoing gravity wave packets as initial perturbations which take the Gaussian forms, we study the effects of nonlinearity on gravity wave packets and focus on the examination of dispersion and polarization relations in the nonlinear propagation of gravity wave packets. The general discussion is given next, and the summary and conclusions are presented in the last section.

\section{Numerical model}

\subsection{Governing equations}

We start from a hydrodynamic equation in an inviscid, compressible, two-dimensional atmosphere, which may be written as

$\frac{\partial \rho}{\partial t}+\frac{\partial(\rho u)}{\partial x}+\frac{\partial(\rho w)}{\partial z}=0$

$\frac{\partial(\rho u)}{\partial t}+\frac{\partial\left(\rho u^{2}\right)}{\partial x}+\frac{\partial(\rho u w)}{\partial z}+\frac{\partial P}{\partial x}=0$

$\frac{\partial(\rho w)}{\partial t}+\frac{\partial(\rho u w)}{\partial x}+\frac{\partial\left(\rho w^{2}\right)}{\partial z}+\frac{\partial P}{\partial z}+g \rho=0$

$\frac{\partial T}{\partial t}+\frac{\partial(T u)}{\partial x}+\frac{\partial(T w)}{\partial z}-T\left(1-\frac{R}{c_{v}}\right)\left(\frac{\partial u}{\partial x}+\frac{\partial w}{\partial z}\right)=0$

$P=\rho R T$

where $x$ is the horizontal coordinate, $z$ is the vertical coordinate (positive upward); $u$ and $w$ are the horizontal and vertical component of total wind velocities, respectively; $\rho, P$ and $T$ are density, pressure and temperature, respectively; $R$ is the universal gas constant $\left(R=286.9821 \mathrm{~J} \mathrm{~kg}^{-1} \mathrm{~K}^{-1}\right) ; c_{v}$ is the specific heat at constant volume $\left(c_{v}=718 \mathrm{~J} \mathrm{~kg}^{-1} \mathrm{~K}^{-1}\right)$; and $g$ is the gravitational acceleration $\left(g=9.80225 \mathrm{~m} \mathrm{~s}^{-2}\right)$.

\subsection{Initial background}

Assuming the initial background is an isothermal atmosphere in hydrostatic equilibrium that is horizontally homogeneous; radar measurements (Tsuda et al., 1990) have shown that mean zonal winds in the range of 60-90 km altitude are $0-40 \mathrm{~m} \mathrm{~s}^{-1}$, so the initial values of horizontal and vertical wind velocities in this study are assumed to be 10 and $0 \mathrm{~m} \mathrm{~s}^{-1}$, respectively. Thus we have

$$
\begin{aligned}
& T_{0}=T_{c} \quad \rho_{0}=\rho_{c} \exp \left(\frac{g}{R T_{c}} z\right) \quad \frac{\partial P_{0}}{\partial z}=-\rho_{0} g \\
& u_{0}=10 \mathrm{~m} \mathrm{~s}^{-1} \quad w_{0}=0 \mathrm{~m} \mathrm{~s}^{-1}
\end{aligned}
$$

where $T_{c}$ and $\rho_{c}$ are constants.

\subsection{Initial perturbation}

An upgoing Gaussian gravity wave-packet is specified as an initial perturbation, thus its horizontal perturbation velocity has the following form:

$$
\begin{aligned}
u^{\prime}(x, z, 0)= & u_{c} \exp \left(-\frac{\left(x-x_{c}\right)^{2}}{2 \sigma_{x}^{2}}\right) \exp \left(-\frac{\left(z-z_{c}\right)^{2}}{2 \sigma_{z}^{2}}\right) \\
& \times \sin \left[k_{x}\left(x-x_{c}\right)+k_{z}\left(z-z_{c}\right)\right]
\end{aligned}
$$

where $u_{c}=1 \mathrm{~m} \mathrm{~s}^{-1} ; x_{c}=1000 \mathrm{~km} ; z_{c}=60 \mathrm{~km} ; k_{x}$ and $k_{z}$ are the horizontal and vertical components of the wave number vector, respectively. $\sigma_{x}$ and $\sigma_{z}$ are the halfwidths of wave packet in the horizontal and vertical direction, respectively. According to the observations at the mesospheric altitudes, the values of the horizontal wavelength $\lambda_{x}$ and the vertical wavelength $\lambda_{z}$ are chosen to be 200 and $10 \mathrm{~km}$, respectively (the initial horizontal phase velocity of this gravity wave is $38.83 \mathrm{~m} \mathrm{~s}^{-1}$ ), and $\sigma_{x}=\lambda_{x}, \sigma_{z}=\lambda_{z}$

The other initial perturbation quantities (i.e. $w^{\prime}(x, z, 0)$, $\left.\rho^{\prime}(x, z, 0), T^{\prime}(x, z, 0), P^{\prime}(x, z, 0)\right)$ are derived from the polarization equations of gravity waves. An arithmetic sum of the initial perturbations and the initial background quantities is regarded as the initial state of Eq. (1).

\subsection{Computational method}

The FICE scheme is applied in the present numerical simulation because of its excellent stability. In order to retain the suitable computational accuracy and avoid the checkerboard solution of pressure and velocity, a uniform Eulerian mesh with a staggered grid is adopted in the calculation domain. In this simulation the horizontal and vertical space step lengths $\Delta x$ and $\Delta z$ are set to be one tenth of $\lambda_{x}$ and $\lambda_{z}$, respectively. The computational method of solving Eq. (1) has been described in detail by $\mathrm{Hu}$ and $\mathrm{Wu}$ (1984).

\subsection{Boundary conditions}

The projected characteristic line boundaries are employed at all boundaries (the top, bottom and lateral 
boundaries). They consist of the compatibility equations along the outgoing projected characteristic lines and the projected characteristic quantity conservation equations along the incoming projected characteristic lines. Detailed account and discussion of the specification of the projected characteristic line boundaries is described by $\mathrm{Hu}$ and Wu (1984) and Zhang and Yi (1999).

\subsection{Time step-length}

A full-implicit scheme should be unconditionally stable. The only stability restriction on the time step-length $\Delta t$ comes from the explicit scheme applied in the specification of boundary conditions (Zhang and Yi, 1999), therefore the stability condition is:

$\Delta t<\Delta t_{c}=\frac{1}{\left(v_{a}+v\right)\left(\frac{1}{\Delta x^{2}}+\frac{1}{\Delta z^{2}}\right)^{\frac{1}{2}}}$

where $v_{a}=\sqrt{\gamma R T}\left(\gamma=c_{p} / c_{v}\right)$ is the acoustic velocity; $v=\sqrt{u^{2}+w^{2}}$. In practical calculation, the shorter time step-length leads to more accurate results, and a shorter time step-length requires fewer iterations for convergence under the same tolerance error. Thus the computational quantities will not increase very much because of the reduction of time step length $(\mathrm{Hu}$ and $\mathrm{Wu}, 1984)$. In this work, we have chosen $\Delta t=0.25 \Delta t_{c}$.

\section{Analyses of the simulation results}

\subsection{Horizontal disturbance velocity}

Figure 1 shows the nonlinear propagation and evolution of the gravity wave packet. The upward propagation of wave packet is clearly shown in Fig. 1. The horizontal perturbation velocity is calculated from the total horizontal wind by applying a high-pass filter, according to the wavelength initially given, the horizontal and vertical cutoff wavelength is $400 \mathrm{~km}$ and $20 \mathrm{~km}$, respectively. In Fig. 1 it can be seen that the gravity wave packet can keep its shape well after a long time (several periods) of propagation, and during the propagation the waveassociated perturbation velocity increases with increasing height. At the beginning, the maximum horizontal perturbation velocity of the wave packet is $1 \mathrm{~m} \mathrm{~s}^{-1}$, during the last several hours of propagation, the amplitude of the horizontal disturbance velocity is much larger than that initially given, after $12 \mathrm{~h}$ of propagation, when the wave packet arrives at heights $\sim 110 \mathrm{~km}$, the horizontal perturbation velocity increases to $\sim 20 \mathrm{~m} \mathrm{~s}^{-1}$. At that height, the horizontal phase velocity is calculated to be $33.33 \mathrm{~m} \mathrm{~s}^{-1}$, while the background wind (specified from the total wind velocity and the disturbance wind velocity) is altered to be about $13 \mathrm{~m} \mathrm{~s}^{-1}$. Thus, the amplitude of the horizontal perturbation velocity divided by intrinsic phase velocity is 0.98 , is slightly less than the instability amplitude (the ratio is 1), which implies that the nonlinearity is significant although far away from the strong nonlinearity.

\subsection{Variation of wavelength}

To study the variation of wavelength of the wave packet in nonlinear propagation, we analyze the wave number spectrum of the horizontal disturbance velocity. Figure 2 is the normalized wave number spectrum of the horizontal disturbance velocity that is shown in Fig. 1. The contour interval is 0.1 , the minimum value is 0.1 , the negative values of $k_{z}$ indicate the phase front motion is downward. It can be observed from Fig. 2 that during the $12 \mathrm{~h}$ of propagation the region in spectral space covered by the gravity wave packet is larger than that given initially, especially in the vertical direction, which is similar to the results presented by Hines (1991). In the horizontal direction the dominant disturbance scale is $200 \mathrm{~km}$ during the whole propagation and has not any evident changes. However, in the vertical direction the dominant disturbance scales decrease clearly, after $4 \mathrm{~h}$ of propagation the dominant vertical disturbance scale decrease from $10 \mathrm{~km}$ (given initially) to $\sim 8.3 \mathrm{~km}$. These results indicate that the nonlinearity will enrich the wave number spectral components of gravity wave disturbance and cause the exchange of wave-associated energies among different disturbance scales.

\subsection{Dispersion relation}

The dispersion relation of gravity waves describes the temporal and spatial evolution of gravity waves, it is the fundamental equation in describing gravity-wave motions. In an isothermal atmosphere the dispersion relation of internal gravity waves (Hines, 1960) is

$k_{x}^{2}\left(1-\frac{\omega_{b}^{2}}{\Omega^{2}}\right)+k_{z}^{2}=\left(\frac{\Omega}{v_{a}}\right)^{2}\left(1-\frac{\omega_{a}^{2}}{\Omega^{2}}\right)$

where $\omega_{a}=\gamma g / 2 v_{a}$ is the acoustic cutoff frequency; $\omega_{b}=\sqrt{\gamma-1}\left(g / v_{a}\right)$ is the buoyancy frequency; $\Omega$ is the intrinsic frequency of a gravity wave and specified by

$\Omega=\omega-k_{x} \bar{u}-k_{z} \bar{w}$

where $\omega$ is the frequency of the gravity wave; $\bar{u}$ and $\bar{w}$ are the horizontal and vertical components of the ambient wind, respectively. Equation (4) gives the well-known dispersion relation of gravity waves under linear conditions, however, in many cases this relation is arbitrarily applied to study gravity wave-associated disturbance in nonlinear circumstance, so it is necessary to compare the frequencies of gravity-wave in nonlinear propagation with the values derived from Eq. (4).

Figure 3 is the frequency variation with time. The solid line denotes the frequencies (ground-based) of gravity wave packet in nonlinear propagation which are calculated in this way: at first we specify the centre positions of the wave packet at each integer hour from the peak of averaged wave energy density (Zhang and $\mathrm{Yi}$, 1999), then we have 13 centre positions for 13 integer hours. For each integer hour, we start at $1 \mathrm{~h}$ before and end at $1 \mathrm{~h}$ after each integer hour except for the 0 (the frequency is given initially) and last hour (10 h to $12 \mathrm{~h}$ ), we record a $2 \mathrm{~h}$ long temporal series of the horizontal 

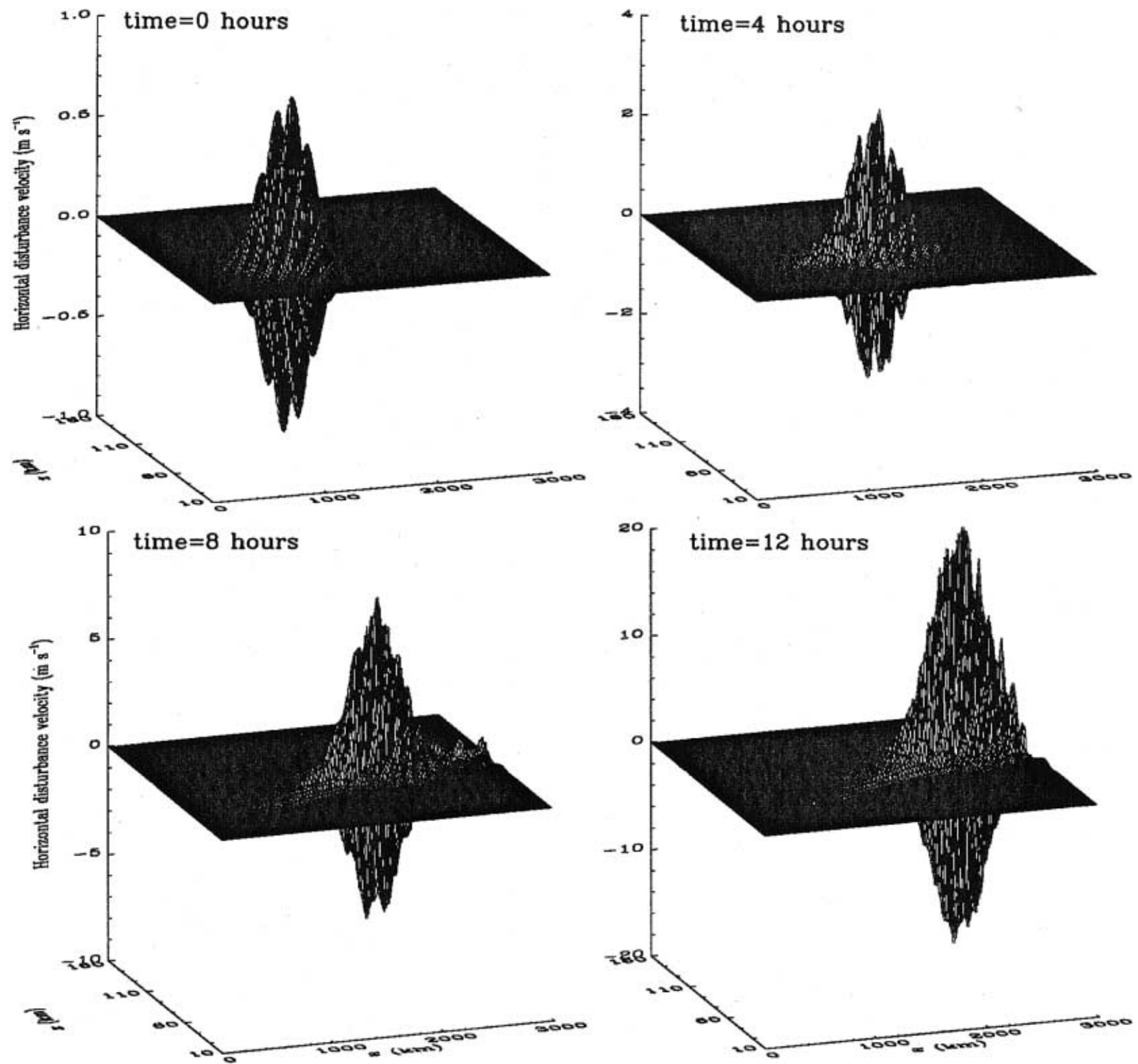

Fig. 1. Propagation of horizontal perturbation velocity of gravity wave packet
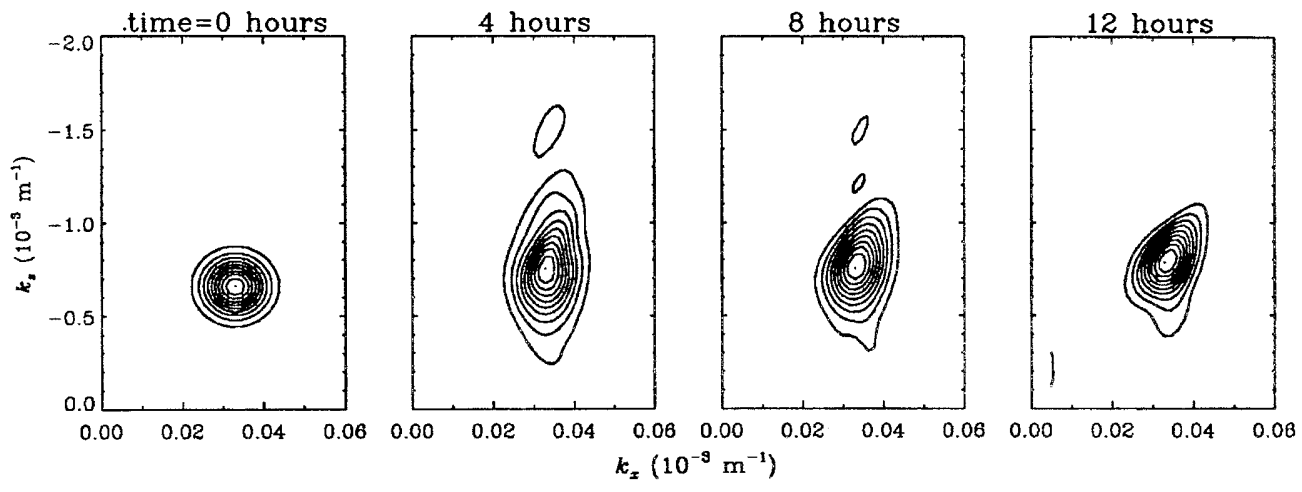

Fig. 2. Normalized wave number spectrum of horizontal perturbation velocity of gravity wave packet 
disturbance velocities at each corresponding centre position of the wave packet, and then make discrete Fourier transformations (DFTs) for each series. Finally we take the frequencies corresponding to the maximum values of the DFTs as the frequencies of the gravity wave packet in nonlinear propagation at each integer hour; the dashed line denotes the frequencies (ground-based) of gravity wave derived from the dispersion relation of gravity wave, e.g. Eqs. (4) and (5). It should be noted that in resolving Eqs. (4) and (5), the values of $k_{x}$ and $k_{z}$ are chosen to be the dominant horizontal and vertical wave numbers at each integer hour (shown in Fig. 2), respectively; the values $\bar{u}, \bar{w}$ and $v_{a}$ are chosen to be the values at the centre of the wave packet at each integer hour. Figure 3 shows that the ground-based nonlinear frequency decreases from $\sim 1.22 \times 10^{-3} \mathrm{rad} \mathrm{s}^{-1}$ at the beginning to $\sim 1.10 \times 10^{-3} \mathrm{rad} \mathrm{s}^{-1}$ after several hours of propagation, this obviously due to nonlinearity or (and) the variation of background with time; moreover, the frequencies (ground-based) derived from the linear gravity wave theory are close to the nonlinear frequency (ground-based) even when the wave amplitude is large, the maximum deviation between them is no more than $0.05 \times 10^{-3} \mathrm{rad} \mathrm{s}^{-1}$, which demonstrates that the dispersion relation based essentially on the linear gravity wave theory is feasible in nonlinear circumstance.

\subsection{Polarization relation}

The polarization relation is the relation among each disturbance components of wave motion on two aspects:

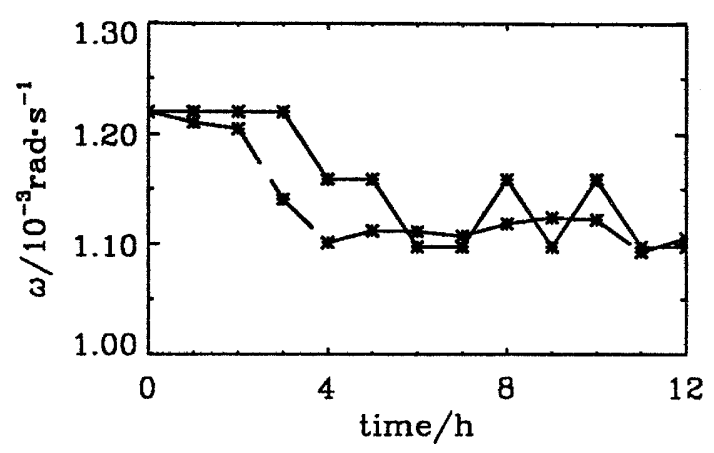

Fig. 3. Frequencies of gravity wave packet amplitudes and phases. Now we will examine whether the polarization relation of the gravity wave is feasible in the nonlinear propagation of gravity waves or not. Since the gravity wave packet can keep its shape very well after a long time nonlinear propagation (shown in Fig. 1), therefore we assume that at each time the timevarying disturbance quantities $X^{\prime}(x, z)\left(u^{\prime}(x, z), w^{\prime}(x, z)\right.$, $\left(\rho^{\prime} / \rho_{0}\right)(x, z)$ and $\left.\left(P^{\prime} / \rho_{0}\right)(x, z)\right)$ satisfy the following spatial distribution:

$$
\begin{aligned}
X^{\prime}= & \exp \left(-\frac{\left(x-x_{m}\right)^{2}}{2 \sigma_{x}^{2}}-\frac{\left(z-z_{m}\right)^{2}}{2 \sigma_{z}^{2}}\right) \\
& \times\left[a \sin \left(k_{x} x+k_{z} z\right)+b \cos \left(k_{x} x+k_{z} z\right)\right]
\end{aligned}
$$

where $x_{m}$ and $z_{m}$ are the centre positions of the wave packet in the $x$ and $z$ direction, respectively. The coefficients $a$ and $b$ are calculated by fitting the simulation results for Eq. (6) using the least square deviation criterion, and then the amplitudes and phases of the disturbance quantities $X^{\prime}$ in nonlinear propagation are determined.

Figure 4 is the amplitude variations of wave-associated disturbance quantities with time. The dotted line in the left panel denotes the amplitude of horizontal disturbance velocity derived from the linear gravity wave theory; the solid lines represent the amplitudes in nonlinear propagation, which are calculated from the coefficients $a$ and $b$. The dashed lines represent the amplitudes derived from the polarization relation of gravity wave by using the nonlinear amplitude of horizontal disturbance velocity $u^{\prime}$. We can see from Fig. 4 that the nonlinear wave-associated disturbance amplitudes increase with time, and the values of amplitudes are rather close to the prediction of linear gravity wave theory; moreover, the nonlinear amplitudes are very close to that derived from the polarization relation of the linear gravity wave theory even when the disturbance amplitudes are increased to be significant, which demonstrates that the polarization relation of gravity waves based on the linear gravity wave theory can well predict the relations of amplitudes among each of the wave-associated disturbance components in the nonlinear case.

The phase variations of each wave-associated disturbance quantity with time are shown in Fig. 5. The solid lines denote the phases in nonlinear propagation, which
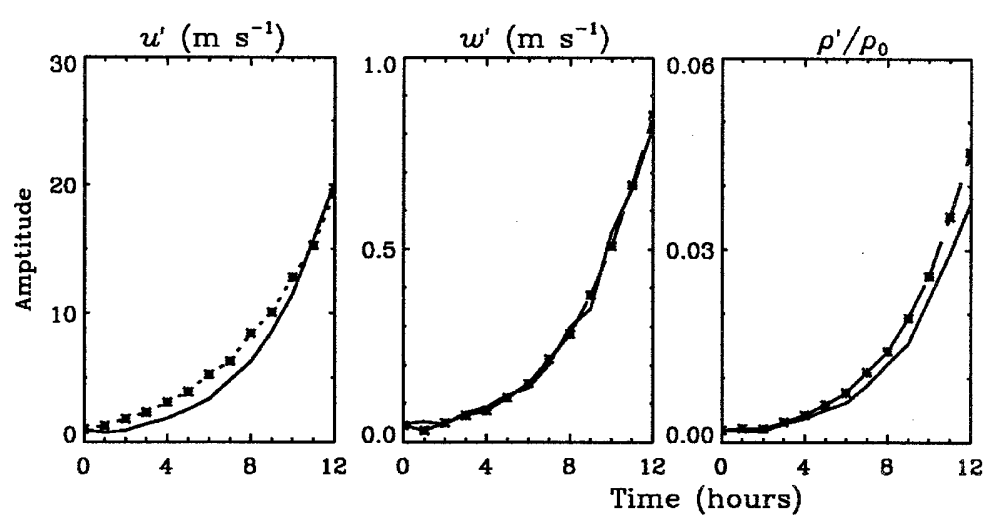

Fig. 4. Amplitudes of gravity wave packet 

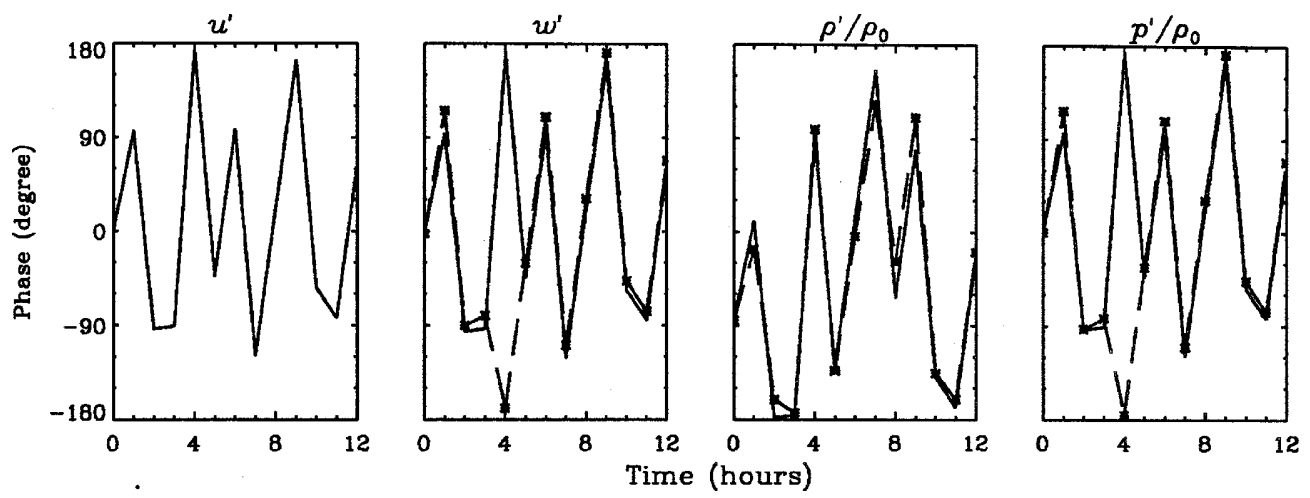

Fig. 5. Phases of gravity wave packet

are calculated from the coefficients $a$ and $b$. The dashed lines denote the phases derived from the polarization relation of gravity waves by using the nonlinear phase of horizontal disturbance velocity $u^{\prime}$. It is clearly shown in Fig. 5 that the nonlinear phases are close to that derived from the polarization relation of gravity wave during the whole propagation of the gravity wave packet, the nonlinearity has little influence on the phases of the wave-associated disturbance quantities. Therefore, combined with the results shown in Fig. 4, we can say that the polarization relation based on the linear gravity wave theory is valid in nonlinear case.

\section{Discussion}

In this section, in order to test the generality of the conclusion drawn in Sect. 3, we simulate the nonlinear propagation of two more gravity wave packets (A and B) with different properties (wavelength, initial amplitude and mean wind, etc.). For wave packet $\mathrm{A}$ the initial horizontal disturbance amplitude and mean wind are 4 and $30 \mathrm{~m} \mathrm{~s}^{-1}$, respectively; the horizontal and vertical wavelengths are 500 and $15 \mathrm{~km}$, respectively, thus the initial horizontal phase velocity is $73.04 \mathrm{~m} \mathrm{~s}^{-1}$. For wave packet $\mathrm{B}$ the initial horizontal disturbance amplitude and mean wind are 2 and $5 \mathrm{~m} \mathrm{~s}^{-1}$, respectively; the horizontal and vertical wavelengths are 100 and $5 \mathrm{~km}$, respectively, thus the initial horizontal phase velocity is $19.46 \mathrm{~m} \mathrm{~s}^{-1}$.

By calculating the wave number spectrum of the horizontal disturbance velocities for wave packets A and B, we attain similar results (shown in Figs. 6 and 7) to that shown in Fig. 2: the region in spectral space covered by the gravity wave packets is larger than that given initially, the dominant disturbance scales in the
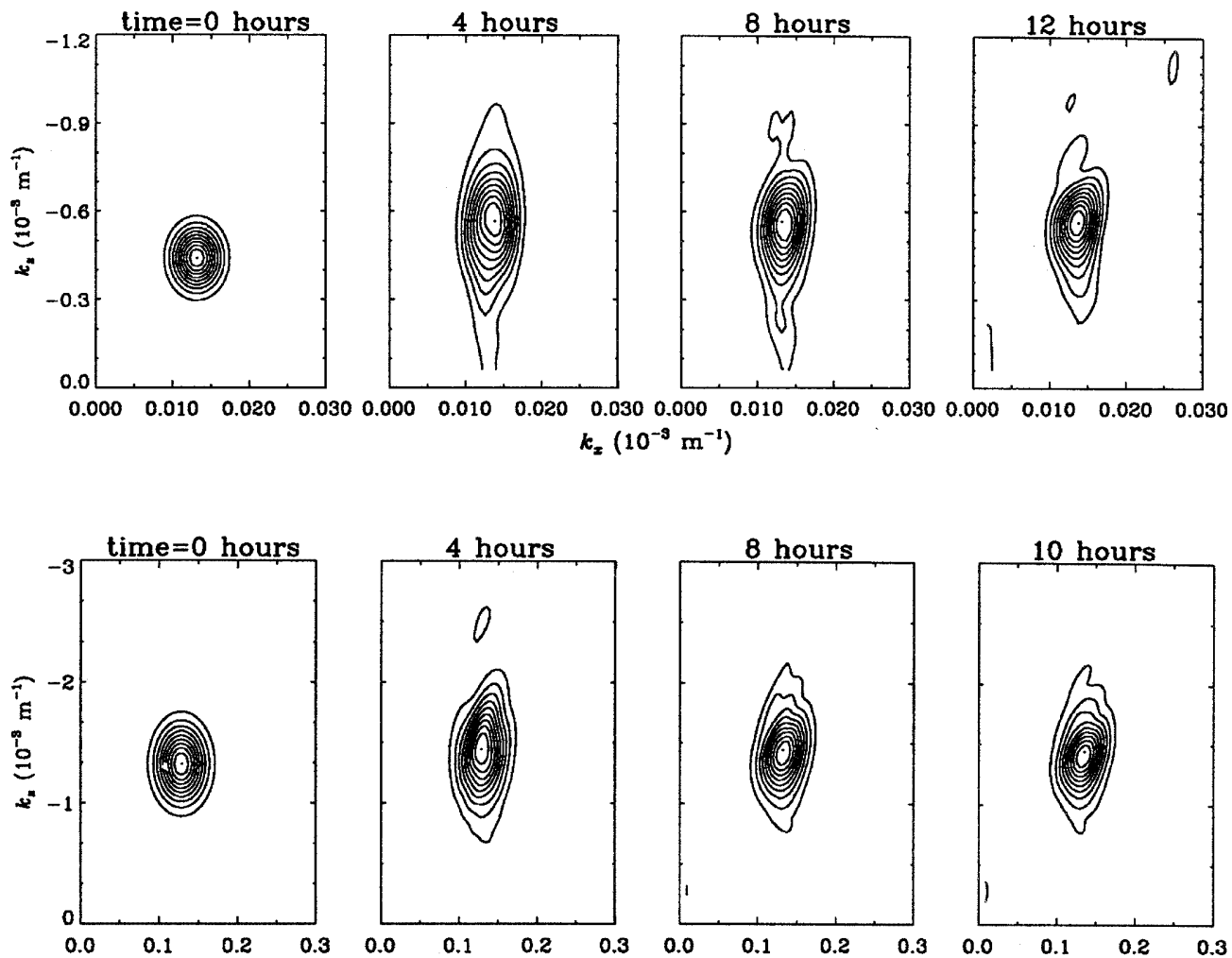

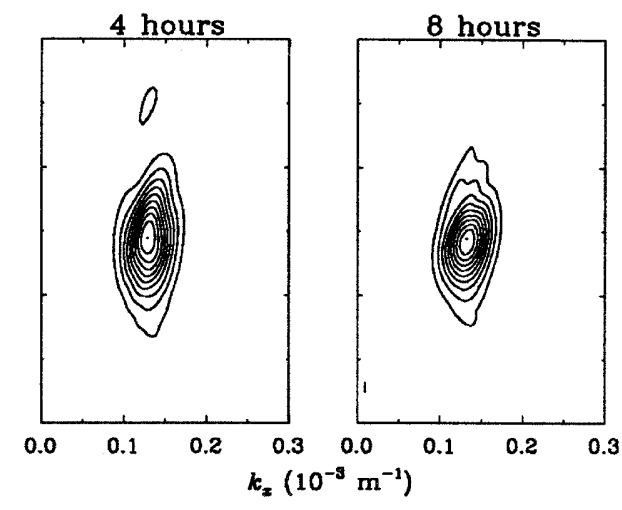

Fig. 6. As for Fig. 2, but for wave packet $\mathrm{A}$

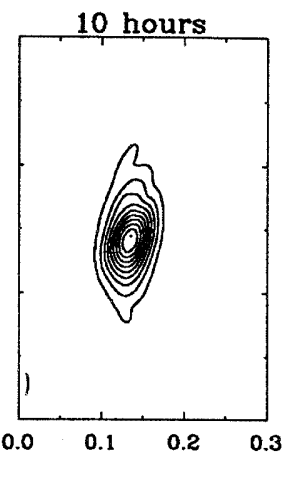

Fig. 7. As for Fig. 2, but for wave packet $\mathrm{B}$ 
horizontal direction do not show evident changes, however, in the vertical direction the dominant disturbance scales clearly decrease. Moreover, the mean flows show enhancement. Thus, it is evident that the nonlinear effects will cause the enhancement of mean flows for a variety of upgoing gravity wave packets propagating in mesosphere.

Figures 8 and 9 are the frequency variations with time for wave packets $\mathrm{A}$ and $\mathrm{B}$, respectively. The solid lines represent the frequencies in nonlinear propagation; the dashed lines represent the frequencies derived from the dispersion relation of gravity wave. These two figures show that nonlinear frequencies decrease with time and frequencies derived from the linear gravity wave theory are close to those nonlinear frequencies. Combined with the result shown in Fig. 3, we can conclude that: for a variety of gravity wave packets, the

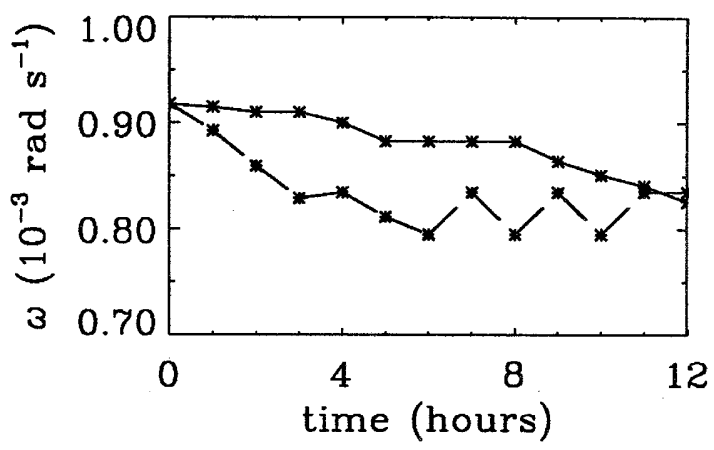

Fig. 8. As for Fig. 3, but for wave packet A

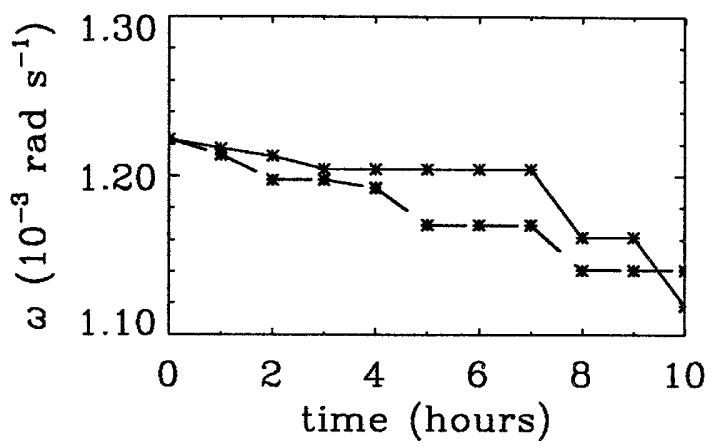

Fig. 9. As for Fig. 3, but for wave packet B dispersion relation based on the line gravity wave theory is feasible in nonlinear circumstance.

Figures 10 and 11 are the amplitudes variations of each disturbance components with time for wave packets A and B, respectively; and Figs. 12 and 13 are the phases variations of each disturbance components with time for waves A and B, respectively. The dotted lines in the left panel of Figs. 10 and 11 denote the amplitudes of horizontal disturbance velocity derived from the linear gravity wave theory; the solid lines denote the amplitudes (phases) in nonlinear propagation and the dashed lines denote the amplitudes (phases) derived from the polarization relation of gravity waves by using the nonlinear amplitudes (phases) of horizontal disturbance velocity $u^{\prime}$. We can see from these figures that both the amplitudes and phases derived from the polarization relation of gravity waves are close to that in nonlinear propagation. Combined with the results shown in Figs. 4 and 5, it can be concluded that: for a variety of gravity wave packets, the polarization relation based on linear gravity wave theory can be applied extensively in nonlinear cases.

\section{Summary and conclusions}

By using a FICE scheme, the nonlinear propagation of several gravity wave packets in a two-dimensional compressible atmosphere has been simulated. The initial perturbations are taken to be upgoing Gaussian gravity wave packets. The initial backgrounds are assumed to be in hydrostatic equilibrium in which the ambient density and pressure decrease exponentially with increasing height. A quantitative comparison of the wave parameters of the gravity wave packets in nonlinear propagation with that derived from the linear theory is presented, and the validity of the dispersion and polarization relations based on linear gravity wave theory in nonlinear cases are also examined. The primary conclusions include the following:

1. For upgoing gravity waves, the nonlinearity will enrich the wave number spectral components of gravity wave-associated disturbance and accelerate the mean flow, which indicate that the nonlinear effects will cause the exchange of wave-associated energies among different disturbance scales.
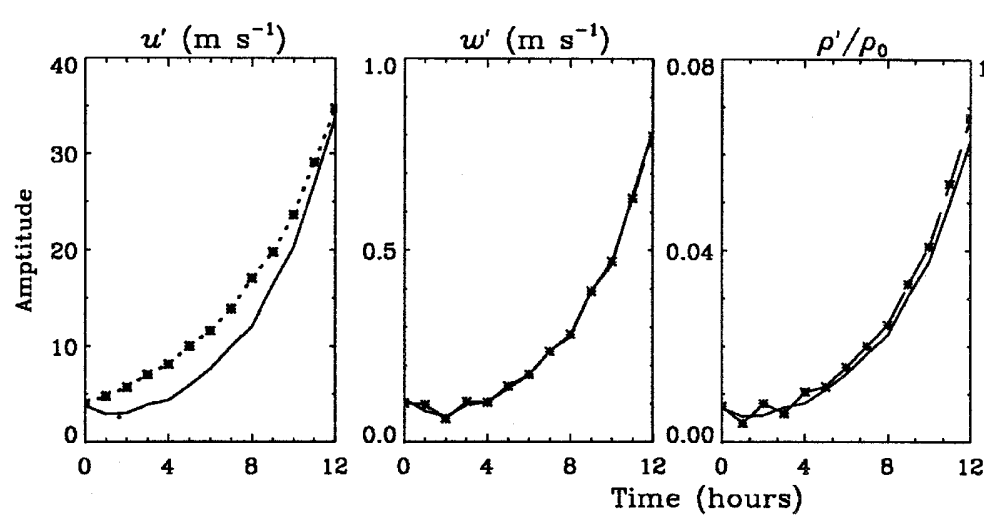

Fig. 10. As for Fig. 4, but for wave packet $A$ 

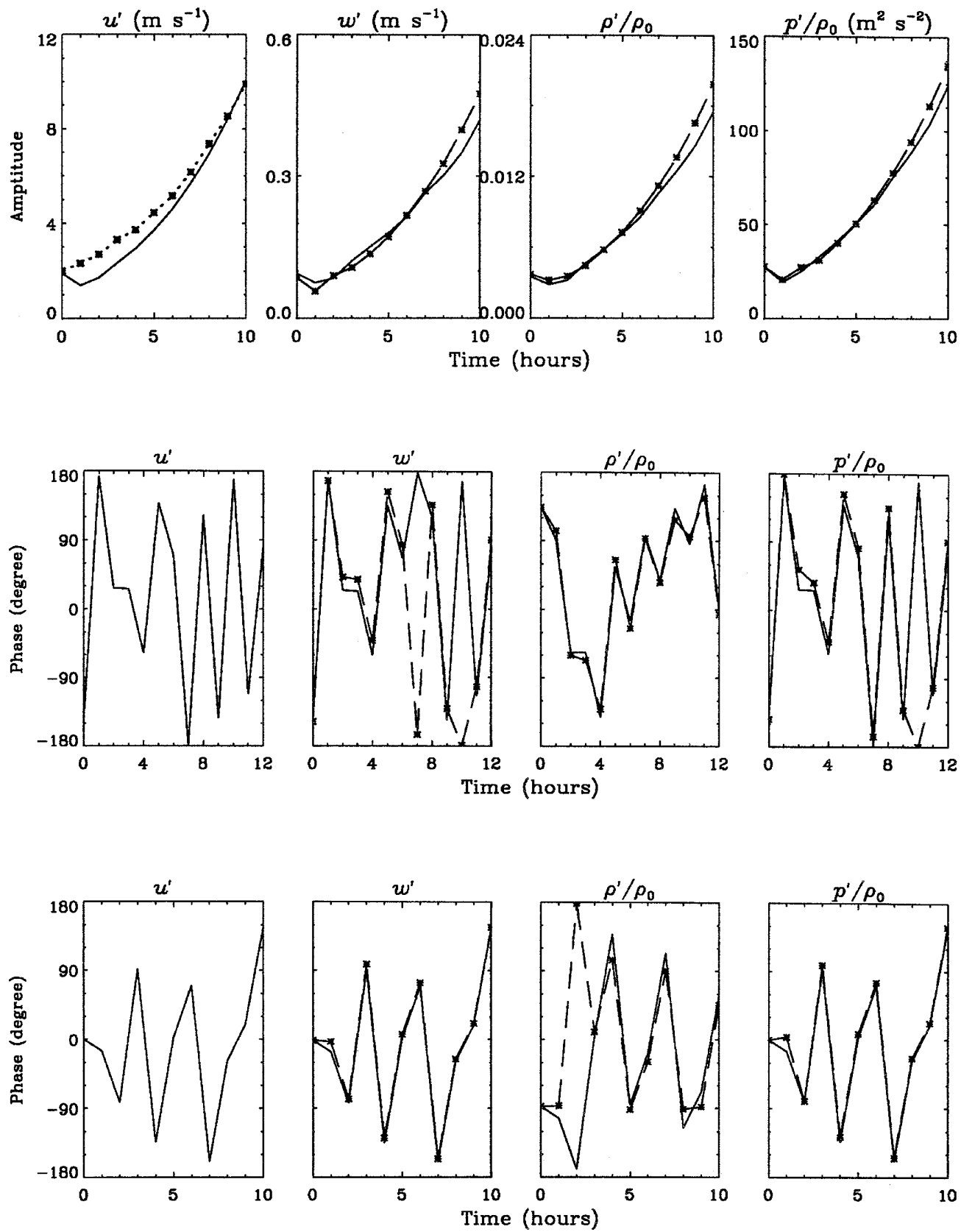

Fig. 11. As for Fig. 4, but for wave packet $B$

Fig. 12. As for Fig. 5, but for wave packet $\mathrm{A}$

Fig. 13. As for Fig. 5, but for wave packet $\mathrm{B}$
2. During the nonlinear propagation of gravity wave packets in isothermal atmosphere, in spite of the slight decrement of frequencies with time due to the nonlinear effects, the nonlinear frequencies are still close to that derived from the dispersion relation, which demonstrates the dispersion relation of gravity waves based essentially on the linear gravity wave theory are feasible in nonlinear regions.

3. Both the time variations of amplitudes and phases of gravity wave packets in nonlinear propagation are close to that derived from the polarization relation of the gravity wave, which demonstrates that the polarization relation of gravity waves can be applied extensively in the nonlinear region, most of the nonlinear kinetical behaviour of gravity waves can be well described by the linear gravity wave theory.
Acknowledgements. We wish to thank two anonymous reviewers for helpful comments and suggestions. This work received support from the National Natural Science Foundations of China through grant 49625407, 49990450 and 49904009. Computer support for the bulk of our calculations was provided by the Ziqiang Foundation of Wuhan University.

Topical Editor D. Murtagh thanks two referees for their help in evaluating this paper.

\section{References}

Dewan, E. M., and R. E. Good, Saturation and the "universal" spectrum for vertical profiles of horizontal scalar winds in the atmosphere, J. Geophys. Res., 91, 2742-2748, 1986.

Fritts, D. C., Gravity waves saturation in the middle atmosphere: A review of theory and observations, Rev. Geophys., 22, 275-308, 1984. 
Fritts, D. C., and P. K. Rastogi, Convective and dynamical instabilities due to gravity wave motions in the lower and middle atmosphere: theory and observations, Radio. Sci., 20, 1247-1277, 1985

Gardner, C. S., Diffusive filtering theory of gravity wave spectra in the atmosphere, J. Geophys. Res., 99, 20 601-20 622, 1994.

Hines, C. O., Internal atmospheric gravity waves at ionospheric heights, Can. J. Phys., 38, 1441-1481, 1960.

Hines, C. O., The Saturation of gravity waves in the middle atmosphere. Part II: development of Doppler-spread theory, J. Atmos. Sci., 48, 1360-1379, 1991.

Holton, J. R., The role of gravity wave-induced drag and diffusion in the momentum budget of the mesosphere, J. Atmos. Sci., 39, 791-799, 1982.

Holton, J. R., The influence of gravity wave breaking on the general circulation of the middle atmosphere, J. Atmos. Sci., 40, 2497-2507, 1983

Hu, Y. Q., and S. T. Wu, A full-implicit-continus-Eulerian (FICE) scheme for multidimensional transient magnetohydrodynamic (MHD), J. Comput. Phys., 55, 33-64, 1984.

Lindzen, R. S., Turbulence and stress owing to gravity wave and tidal breakdown, J. Geophys. Res., 86, 9707-9714, 1981.

Manson, A. H., Gravity wave horizontal and vertical wave-length: an update of measurements in the mesopause region $(\sim 80$ $100 \mathrm{~km})$, J. Atmos. Sci., 47, 2765-2773, 1990.

Meek, C. E., I. M. Reid, and A. H. Manson, Observations of mesospheric wind velocities, 1, Gravity wave horizontal scales and phase velocities from spaced wind observations, Radio Sci., 20, 1363-1382, 1985.

Mobbs, S. D., A numerical investigation of nonlinear internal gravity waves and their influence on the mean flow, Ann. Geophysicae, 5, 197-208, 1987.

Prusa, J. M., Propagation and breaking at high altitudes of gravity waves excited by tropospheric forcing, J. Atmos. Sci., 53, 21862216, 1996.

Tsuda, T., S. Kato, T. Yokoi, T. Inoue, and M. Yamamoto, Gravity waves in the mesosphere observed with the middle and upper atmosphere radar, Radio Sci., 26, 1005-1018, 1990.

Vincent, R. A., Gravity wave motions in the mesosphere and lower thermosphere observed at Mawson, Antarctica, J. Atmos. Sol. Terr. Phys., 56, 593-602, 1994.

Vincent, R. A., and D. C. Fritts, A climatology of gravity wave motions in the mesopause region at Adelaide, Australia, J. Atmos. Sci., 44, 748-760, 1987.

Walterscheid, R. L., and G. Schubert, Nonlinear evolution of an upward propagation gravity wave: overturning, convection, transience and turbulence, J. Atmos. Sci., 47, 101-125, 1990.

Yamamoto, M., T. Tsuda, and S. Kato, Gravity waves observed by the Kyoto meteor radar in 1983-1985, J. Atmos. Sol. Terr. Phys., 48, 597-603, 1986.

Yeh, K. C., and C. H. Liu, Acoustic-gravity waves in the upper atmosphere, Res. Geophys., 12, 193-216, 1974.

Zhang, S. D., and F. Yi, A numerical study of nonlinear propagation of a gravity-wave packet in compressible atmosphere. J. Geophys. Res., 104, 14 261-14 270, 1999. 